\title{
An extremely prolific supernova factory in the buried nucleus of the starburst galaxy IC $694^{\star}$
}

\author{
M. A. Pérez-Torres ${ }^{1}$, C. Romero-Cañizales ${ }^{1}$, A. Alberdi ${ }^{1}$, and A. Polatidis ${ }^{2,3}$ \\ ${ }^{1}$ Instituto de Astrofísica de Andalucía - CSIC, PO Box 3004, 18008 Granada, Spain \\ e-mail: torres@iaa.es \\ 2 Joint Institute for VLBI in Europe (JIVE), Dwingeloo, The Netherlands \\ 3 ASTRON, Dwingeloo, The Netherlands
}

Received 23 July 2009 / Accepted 17 October 2009

\section{ABSTRACT}

\begin{abstract}
Context. The central kiloparsec of many local uminous infrared galaxies are known to host intense bursts of massive star formation, leading to numerous explosions of core-collapse supernovae (CCSNe). However, the dust-enshrouded regions where those supernovae explode hamper their detection at optical and near-infrared wavelengths.

Aims. We investigate the nuclear region of the starburst galaxy IC 694 (=Arp 299-A) at radio wavelengths, aimed at discovering recently exploded CCSNe, as well as determining their rate of explosion, which carries crucial information about star formation rates, the initial mass function, and the starburst processes in action.

Methods. We use the electronic European VLBI Network to image with milliarcsecond resolution the $5.0 \mathrm{GHz}$ compact radio emission of the innermost nuclear region of IC 694.

Results. Our observations detect a rich cluster of 26 compact radio emitting sources in the central $150 \mathrm{pc}$ of the nuclear starburst in IC 694. The high brightness temperatures observed for the compact sources are indicative of a non-thermal origin for the observed radio emission, implying that most, if not all, of those sources are young radio supernovae (RSNe) and supernova remnants (SNRs). We find evidence of at least three relatively young, slowly evolving, long-lasting RSNe (A0, A12, and A15) that appear to have unusual properties, suggesting that the conditions in the local circumstellar medium (CSM) play a significant role in determining the radio behaviour of expanding SNe. Their radio luminosities are typical of normal RSNe, which result from the explosion of type IIP/b and type IIL SNe. All of these results provide support for a recent (less than 10-15 Myr) instantaneous starburst in the innermost regions of IC 694.
\end{abstract}

Key words. galaxies: starburst - stars: supernovae: general - radiation mechanisms: non-thermal - radio continuum: stars galaxies: individual: IC 694 - galaxies: luminosity function, mass function

\section{Introduction}

The observed rate at which massive stars $\left(M \gtrsim 8 M_{\odot}\right)$ die as $\mathrm{CCSNe}, v_{\mathrm{CCSN}}$, can be used as a direct measurement of the current star formation rate (SFR) in galaxies, and provides unique information about the initial mass function (IMF) of massive stars. While the rate at which stars die in normal galaxies is rather low (e.g., one $\mathrm{SN}$ is expected to explode in the Milky Way every $\sim 50 \mathrm{yr}$ ), the CCSN rate in luminous and ultraluminous infra-red galaxies (LIRGs, $L_{\mathrm{IR}} \equiv L[8-1000 \mu \mathrm{m}] \geq 10^{11} L_{\odot}$; and ULIRGs, $L_{\mathrm{IR}} \geq 10^{12} L_{\odot}$; Sanders \& Mirabel 1996) is expected to be at least one or two orders of magnitude higher than in normal galaxies (Condon 1992), and hence detections of SNe in (U)LIRGs offer a promising way of determining the current star formation rate in nearby galaxies.

However, the direct detection of CCSNe in the extreme densities of the central few hundred pc of (U)LIRGs is extremely difficult, since emission in the visual band suffers very significant extinction in those regions, which contain large amounts of dust, and can at best yield only an upper limit to the true value of $v_{\mathrm{CCSN}}$. Fortunately, it is possible to directly probe the star-forming activity in the innermost regions of (U)LIRGs by

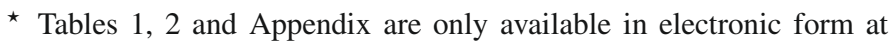
http://www . aanda. org means of high angular resolution ( $\leq 0.05$ arcsec), high-sensitivity $(\leq 0.05 \mathrm{mJy})$ radio searches of CCSNe, as radio emission is unaffected by dust extinction, and the angular resolution yielded by current Very Long Baseline Interferometry (VLBI) arrays, of the order of a few milliarcsec at cm-wavelengths, is able to detect individual radio supernovae at large distances in the local Universe.

Starburst activity in the circumnuclear regions of (U)LIRGs implies both the presence of a high number of massive stars and a dense surrounding medium, so bright radio $\mathrm{SNe}$ are expected to occur (Chevalier 1982; Chugai 1997), and high-resolution radio observations have shown that highly extinguished CCSNe do exist in the circumnuclear ( $r \lesssim 1 \mathrm{kpc}$ ) region of local (U)LIRGs (Smith et al. 1998; Lonsdale et al. 2006; Colina et al. 2001; Neff et al. 2004; Pérez-Torres et al. 2007; Kankare et al. 2008). Therefore, VLBI observations can place strong constraints on the properties of star formation in the dust-enshrouded environments encountered in (U)LIRGs.

Arp 299 consists of two interacting galaxies (IC 694 and NGC 3690), which are in an early merger stage (Keel \& Wu 1995). At a luminosity distance of $44.8 \mathrm{Mpc}$ (Fixsen et al. 1996) for $H_{0}=73 \mathrm{~km} \mathrm{~s}^{-1} \mathrm{Mpc}^{-1}$, Arp 299 has an infrared luminosity $L_{\mathrm{IR}} \approx 6.7 \times 10^{11} L_{\odot}$ (Sanders et al. 2003), which almost qualifies it as a ULIRG. The innermost $\sim 150 \mathrm{pc}$ nuclear 
region of Arp 299-A (see Fig. 1) is heavily dust-enshrouded, thus making the detections of SNe very challenging even at nearinfrared wavelengths. Yet, Arp 299 hosts recent and intense starforming activity, as indicated by the relatively high frequency of supernovae discovered at optical and near-infrared wavelengths in its outer, much less extinguished regions (Forti et al. 1993; van Buren et al. 1994; Li et al. 1998; Yamaoka et al. 1998; Qiu et al. 1999; Mattila et al. 2005).

The brightest component at infrared and radio wavelengths is IC 694 (A in the top panel of Fig. 1; hereafter Arp 299-A), which accounts for $\sim 50 \%$ of the total infrared luminosity of the system (Alonso-Herrero et al. 2000; Charmandaris et al. 2002), and $\sim 70 \%$ of its $5 \mathrm{GHz}$ radio emission (Neff et al. 2004). Numerous $\mathrm{H}$ II regions populate the system near star-forming regions, which implies that star formation has been occurring at a high rate for past $\sim 10 \mathrm{Myr}$ (Alonso-Herrero et al. 2000). Given that IC 694 accounts for most of the infrared emission in Arp 299, it is the region that is most likely to contain new $\mathrm{SNe}$ (Condon 1992). Since optical and near-infrared observations are likely to miss a significant fraction of $\mathrm{CCSNe}$ in the innermost regions of Arp 299-A due to high values of extinction $\left(A_{V} \sim 34-40\right.$, Gallais et al. 2004; Alonso-Herrero et al. 2009) and the lack of the necessary angular resolution, radio observations of Arp 299A at high angular resolution, high sensitivity are the only way of detecting new CCSNe and measuring directly and independently of any model its CCSN and star formation rates. Very Long Baseline Array (VLBA) observations carried out during 2002 and 2003 resulted in the detection of five compact sources (Neff et al. 2004), one of which (A0) was identified as a young SN.

\section{2. eEVN observations and results}

We used the electronic European VLBI Network (e-EVN) (Szomoru 2006) to image Arp 299-A at a frequency of $5 \mathrm{GHz}$ over 2 epochs, to directly detect recently exploded core-collapse supernovae by means of the variability of their compact radio emission (see Appendix A for a detailed description of our observing strategy, calibration and imaging procedures, and source detection and techniques for flux density extraction). The attained off-source root-mean-square (rms) noise level was $39 \mu \mathrm{Jy} /$ beam and $24 \mu \mathrm{Jy} /$ beam for the 8 April 2008 and 5 December 2008 observations, respectively, and enables 26 compact components to be detected above 5 rms (see Fig. 1). Since the EVN radio image on 5 December 2008 is much deeper than the one obtained on 8 April 2008, it is not surprising that we detected a larger number of VLBI sources in our second epoch (25) than in our first one (15). This allowed us to go back to our first-epoch image and extract the flux density for the new components (A15 through to A25 in Fig. 1), which show $\geq 5 \mathrm{rms}$ detections only in the December 2008 image. This procedure allowed us to recover four components above $3 \sigma$ (A15, A18, A22, and A25), based on a positional coincidence with the peak of brightness of our second epoch of greater than $\sim 0.5$ milliarcsec, i.e., much smaller than the synthesized interferometric beam.

Our results demonstrate that a very compact rich nuclear starburst in Arp 299-A exists and, in general, are in excellent agreement with independent results reported by Ulvestad (2009). The angular size encompassed by the radio emitting sources in Arp 299-A is smaller than $0.7^{\prime \prime} \times 0.4^{\prime \prime}$, corresponding to a projected linear size of $(150 \times 85) \mathrm{pc}$. To facilitate comparisons, we define here a fiducial supernova radio luminosity equal to three times the image rms in the 8 April 2008 epoch, which corresponds to $2.9 \times 10^{26} \mathrm{erg} \mathrm{s}^{-1} \mathrm{~Hz}^{-1}$. In this way, the radio luminosities for the VLBI components range between 1.1 (A25)
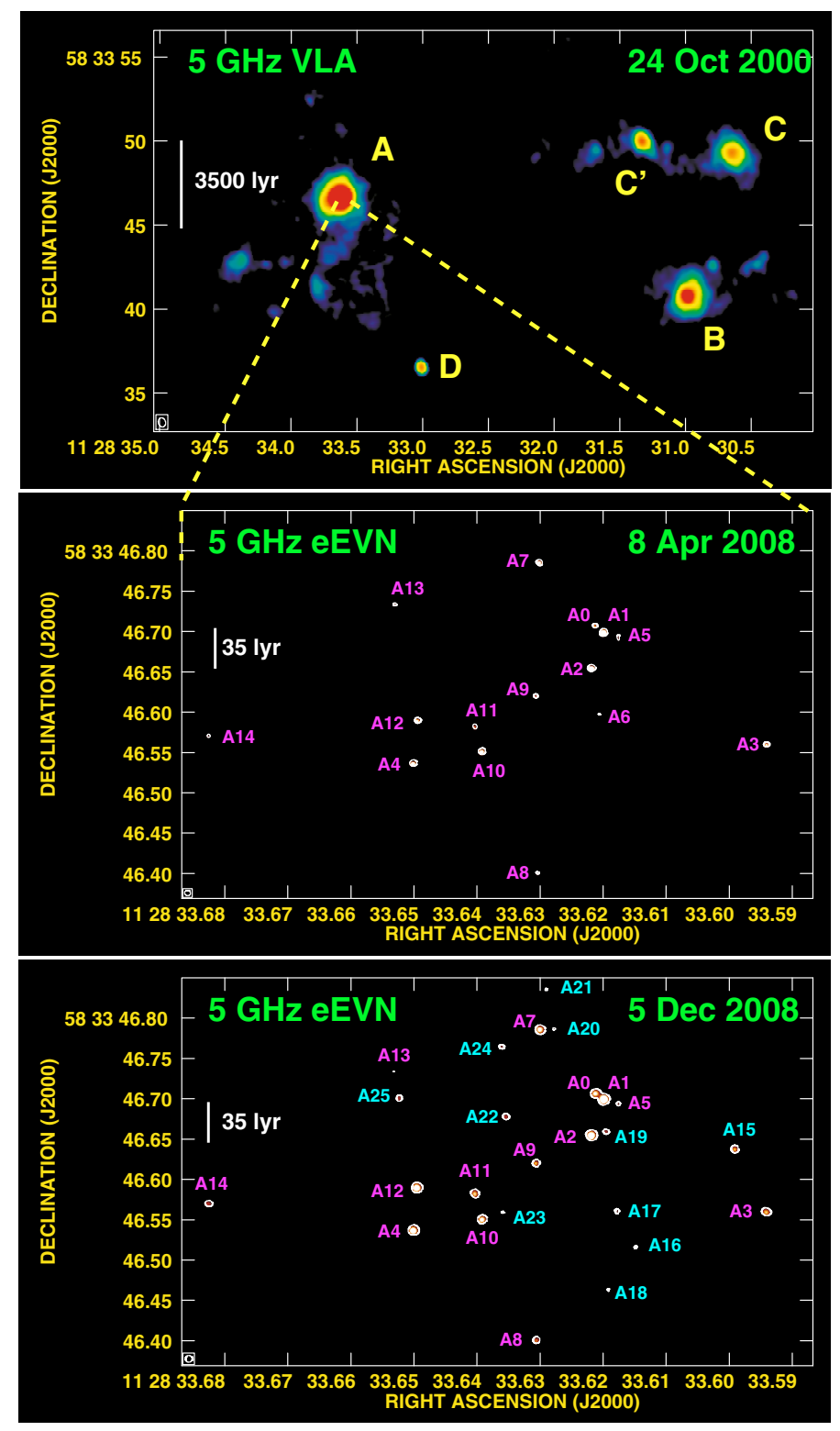

Fig. 1. Top: $5 \mathrm{GHz}$ VLA archival observations of Arp 299 on 24 October 2000, displaying the five brightest knots of radio emission in this merging galaxy. Middle and bottom: contour maps drawn at five times the rms of our $5 \mathrm{GHz}$ eEVN observations of the central 500 light years of the luminous infrared galaxy Arp 299-A on 8 April 2008 and 5 December 2008, revealing a large population of relatively bright, compact, non-thermal emitting sources. The size of the FWHM synthesized interferometric beam was $(0.6 \operatorname{arcsec} \times 0.4 \operatorname{arcsec})$ for the VLA observations, and (7.3 milliarcsec $\times 6.3$ milliarcsec) and (8.6 milliarcsec $\times 8.4$ milliarcsec) for the EVN observations on 8 April 2008 and 5 December 2008, respectively. To guide the reader's eye, we show in cyan the components detected only at the 5 December 2008 epoch.

and 7.3 (A1) and between 1.0 (A13) and 7.7 (A1) times the fiducial value, for the VLBI observations on 8 April 2008 and 5 December 2008, respectively (see Table 1 for details).

\section{Discussion}

The radio emission from the compact sources detected from our VLBI observations can be explained in principle within two different physical scenarios: (i) thermal radio emission from super star clusters (SSCs) hosting large numbers of young, 


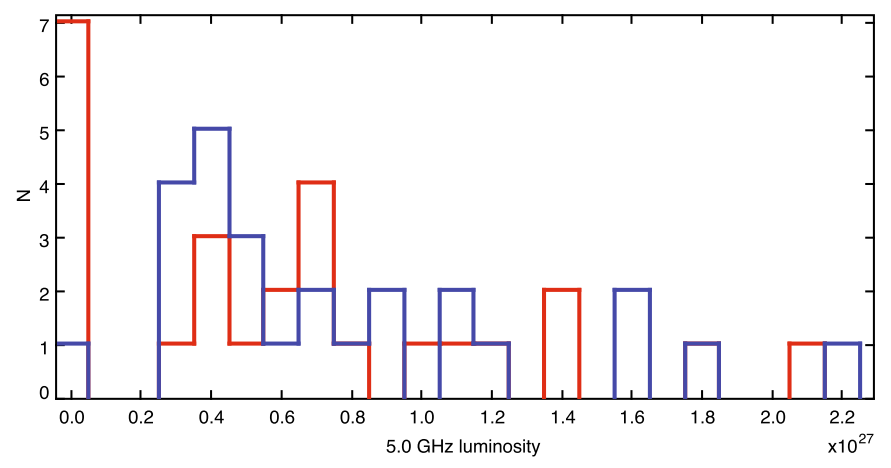

Fig. 2. 5.0 GHz luminosity histogram for the VLBI components detected on 8 April 2008 (red) and 5 December 2008 (blue). The values centered at 0.0 correspond to the upper limits quoted in Table 1 . See main text for details.

massive stars that ionize surrounding H II regions; (ii) nonthermal radio emission from supernova remnants (SNRs) and/or young radio supernovae (RSNe), i.e., recently exploded corecollapse supernovae where the interaction of their ejecta with their surrounding circumstellar or interstellar medium (CSM or ISM, respectively) would give rise to significant amounts of synchrotron radio emission.

The existence of SSCs in Arp 299-A has been demonstrated by their apparent detection using 2.2- $\mu \mathrm{m}$ adaptive optics imaging (Lai et al. 1999). Further evidence comes from Hubble Space Telescope (HST) FOC and NICMOS images, which reveal a population of young stellar clusters in the central regions of Arp 299 (Alonso-Herrero et al. 2000). The total 5.0 GHz radio luminosity in compact sources is $1.7 \times 10^{28} \mathrm{erg} \mathrm{s}^{-1} \mathrm{~Hz}^{-1}$ and $2.0 \times 10^{28} \mathrm{erg} \mathrm{s}^{-1} \mathrm{~Hz}^{-1}$ on 8 April 2008 and 5 December 2008, respectively. However, the high flux densities and small sizes of most of the compact sources in Arp 299-A ( $\leq 9$ milliarcsec; see Table 2), are indicative of brightness temperatures, $T_{\mathrm{B}}$, that greatly exceed the thermal temperatures expected from SSCs $\left(\$ 2 \times 10^{4} \mathrm{~K}\right)$, thus ruling out a thermal origin of the compact radio emission traced by our eEVN observations.

Therefore, the observed radio emission must be generated by young radio supernovae, SNRs, or both. In Table 1, we show the flux densities and luminosities of all the components identified in our observations, and classify the objects according to their variability. The majority of sources do not show any evidence of significant variability, which is consistent with their identification as SNR. Only three sources (A6, A12, and A15) show appreciable flux density variations between our two consecutive VLBI observations (see Table 1 for details), which is very difficult to reconcile with their radio emission being produced by SNRs. A6, A12, and A15 are also detected in previous VLBI observations between 2003 and 2005 by Ulvestad (2009), where he reports that he finds no evidence of significant variability at the $\sim 20 \%$ level. However, the individual flux densities at the various epochs of observation are not listed in his paper, which makes a detailed comparison with our data problematical. During 18 months, a recent RSN may have indeed gone from very low to very high flux density values, and even have faded away completely. Nevertheless, from his non-detection of A15 at $2.3 \mathrm{GHz}$, and our clear detection with an increasing flux density over two epochs at $5.0 \mathrm{GHz}$, we suggest that A15 is a relatively recent, slowly evolving RSN. This behaviour is very similar to that displayed by A0 (see below). Similarly, source A12 has a flux that is increasing with time at $5.0 \mathrm{GHz}$; and since it was also previously detected in 2003 and 2005, it too is likely to be a relatively recent and slowly evolving RSN. The nature of A6 is less clear. Ulvestad (2009) detected A6 only at $2.3 \mathrm{GHz}$. This detection, together with the sudden drop in its $5.0 \mathrm{GHz}$ flux density between April and December 2008 may be interpreted as the result of a CCSN leaving its young radio supernova phase (when its radio emission is powered by interaction with the CSM) and entering a upernova remnant phase (when it is powered by interaction with the ISM). We cannot exclude, however, the possibility that A6 is an X-ray binary or a microquasar. A6 is quite close to two X-ray sources reported by Zezas et al. (2003) (sources 14 and 16 in their Table 1). The combined absorption corrected $\mathrm{X}$-ray luminosity of those sources is $\sim 1.9 \times 10^{40} \mathrm{erg} \mathrm{s}^{-1}$. From the $5.0 \mathrm{GHz}$ radio luminosity of A6 in Table 1, and assuming a spectral index of $\alpha=-0.5$ (typical of microquasars), the approximate total radio luminosity is $\sim 4.8 \times 10^{36} \mathrm{erg} \mathrm{s}^{-1}$, resulting in a ratio of radio to $X$ ray luminosity of $\sim 2.5 \times 10^{-4}$, which is quite high, but still compatible with A6 being a microquasar. Approved new EVN high-sensitivity observations will allow us to confirm the nature of $\mathrm{A} 6$ in the near future.

The maximum 5.0 GHz luminosities inferred for A12 and $\mathrm{A} 15$ are $\sim 1.6 \times 10^{27} \mathrm{erg} \mathrm{s}^{-1} \mathrm{~Hz}^{-1}$ and $\sim 7.6 \times 10^{26} \mathrm{erg} \mathrm{s}^{-1} \mathrm{~Hz}^{-1}$, respectively. Those radio luminosities are typical of radio emitting type IIP, or type IIb SNe (Chevalier 2006). If confirmed, it would be the first time that these relatively faint radio SNe have been detected in the nuclear starburst of a local (U)LIRG. For comparison, Arp 220 appears to contain essentially very bright radio supernovae, which are identified with type IIn SNe (Parra et al. 2007). We note here that this is not simply a sensitivity issue, since some of the VLBI observations of Arp 220 had an $\mathrm{rms}$ as low as $9 \mu \mathrm{Jy} / \mathrm{b}$ (Parra et al. 2007), yet most of the objects detected were identified, based on their high radio luminosities, as type IIn SNe. We cannot exclude, however, that A12 and A15 have not yet reached their peak luminosity. In this case, their (peak) radio luminosity should be a few times higher, and given their slow evolution, these SNe could be type IIL, or even type IIn.

In a previous paper, Neff et al. (2004) reported the detection of five VLBI sources (A0 to A4), within the central $\sim 80 \mathrm{pc}$ of Arp 299-A. We detect all of these components at 5.0 GHz. Components A1 to A4 do not exhibit significant variability in the eight months covered by our $5.0 \mathrm{GHz}$ eEVN observations, which is consistent with them being young supernova remants, as also suggested by Ulvestad (2009).

First detected at $8.4 \mathrm{GHz}, \mathrm{A} 0$ was identified with a radio supernova by Neff et al. (2004). We have now detected it at 5.0 GHz, more than five years after its discovery at $8.4 \mathrm{GHz}$. This implies that A0 is a long-lasting, slowly evolving, non-standard radio supernova, since most other examples evolve more rapidly (Weiler et al. 2002). Although rather uncommon, there are similar cases reported in the literature, in both normal galaxies, e.g., SN 1979C in M100 Montes et al. (2000), and (U)LIRGs, like some of the RSNe in Arp 220 (Parra et al. 2007). In addition, its non-detection at $2.3 \mathrm{GHz}$ until 2005 (Ulvestad 2009) is indicative of a foreground absorber (e.g., a nearby H II region), as in the case of SN 2000ft in the LIRG NGC 7469 (Alberdi et al. 2006; Pérez-Torres et al. 2009). Finally, we note that A5 may also have similar properties, although its variability significance is lower. In short, the behaviour displayed by A0 (and A5) whilst unusual, is not unknown for an RSN, and provides important information about how the interaction between the SN and CSM is proceeding, and thus probes the mass-loss history of the progenitor star. We therefore suggest that it is the local CSM conditions that are primarily responsible for determining 
the power of the radio supernovae exploding in the nuclear starburst of Arp 299-A.

e-EVN observations show that Arp 299-A hosts an extremely prolific supernova factory, with radio luminosities typical of type IIb, IIp, and IIL, and provides strong support to the scenario proposed by Alonso-Herrero et al. (2000), which proposes the existence of a recent (less than 10-15 Myr old), intense, instantaneous starburst. We find evidence of at least three slowly evolving, long-lasting, non-standard RSNe (A0, A12, and A15), which is very indicative of local CSM conditions playing a main role in determining the radio behaviour of the exploding $\mathrm{SNe}$. Our current monitoring of Arp 299-A with the eEVN at $5.0 \mathrm{GHz}$, which is scheduled to continue until the end of 2010, should allow us to detect any new radio supernova, and therefore test whether the IMF in Arp 299-A is top-heavy, in contrast with to the conventional Salpeter (Salpeter 1955), or Kroupa (Kroupa 2001) IMFs, where the production of massive stars $\left(M \gtrsim 8 M_{\odot}\right)$ that eventually produce CCSNe is low compared to the production of less massive stars. There seems to be evidence that this might also be the case of M 82 (Doane \& Mathews 1993) and Arp 220 (Parra et al. 2007), and theoretically it is expected that in the warm, dense, ISM conditions within a (U)LIRG, the IMF should indeed be top-heavy because of a higher Jeans mass (Klessen et al. 2007).

Acknowledgements. We are very grateful to the anonymous referee for many suggestions and comments which have significantly improved the science and contents of our paper. We also thank L. Colina, A. Alonso-Herrero, J. M. Torrelles, E. Alfaro, and S. Mattila for many useful comments on the manuscript and insightful discussions. The continuing development of e-VLBI within the EVN is made possible via the EXPReS project funded by the EC FP6 IST Integrated infrastructure initiative contract \#026642 - with a goal to achieve $1 \mathrm{Gbit} / \mathrm{s}$ e-VLBI real time data transfer and correlation. The EVN is a joint facility of European, Chinese, South African and other radio astronomy institutes funded by their national research councils. MAPT, CRC, and AA acknowledge support by the Spanish Ministry of Education and Science (MEC) through grant AYA 2006-14986-C02-01.

\section{References}

Alonso-Herrero, A., Rieke, G. H., Rieke, M. J., \& Scoville, N. Z. 2000, ApJ, 532,845

Alonso-Herrero, A., Rieke, G. H., Colina, L., et al. 2009, ApJ, 697, 660
Beasley, A. J., \& Conway, J. E. 1995, in Very Long Baseline Interferometry and the VLBA, ed. J. A. Zensus, P. J. Diamond, \& P. J. Napier, ASP Conf. Ser., 82,328

Bridle, A. H., \& Schwab, F. R. 1999, in Synthesis Imaging in Radio Astronomy II, ed. G. B. Taylor, C. L. Carilli, \& R. A. Perley, ASP Conf. Ser., 180, 371

Charmandaris, V., Stacey, G. J., \& Gull, G. 2002, ApJ, 571, 282

Chevalier, R. A. 1982, ApJ, 259, 302

Chevalier, R. A. 2006, arXiv Astrophysics e-prints

Chugai, N. N. 1997, Ap\&SS, 252, 225

Colina, L., Alberdi, A., Torrelles, J. M., Panagia, N., \& Wilson, A. S. 2001, ApJ, 553, L19

Condon, J. J. 1992, ARA\&A, 30, 575

Doane, J. S., \& Mathews, W. G. 1993, ApJ, 419, 573

Fixsen, D. J., Cheng, E. S., Gales, J. M., et al. 1996, ApJ, 473, 576

Forti, G., Boattini, A., Tombelli, M., Herbst, W., \& Vinton, G. 1993, IAU Circ., 5719, 3

Gallais, P., Charmandaris, V., Le Floc'h, E., et al. 2004, A\&A, 414, 845

Kankare, E., Mattila, S., Ryder, S., et al. 2008, ApJ, 689, L97

Keel, W. C., \& Wu, W. 1995, AJ, 110, 129

Klessen, R. S., Spaans, M., \& Jappsen, A.-K. 2007, MNRAS, 374, L29

Kroupa, P. 2001, MNRAS, 322, 231

Lai, O., Rouan, D., Rigaut, F., Doyon, R., \& Lacombe, F. 1999, A\&A, 351, 834

Li, W.-D., Li, C., Wan, Z., Filippenko, A. V., \& Moran, E. C. 1998, IAU Circ., 6830,1

Lonsdale, C. J., Diamond, P. J., Thrall, H., Smith, H. E., \& Lonsdale, C. J. 2006, ApJ, 647, 185

Mattila, S., Monard, L. A. G., \& Li, W. 2005, IAU Circ., 8473, 1

Montes, M. J., Weiler, K. W., Van Dyk, S. D., et al. 2000, ApJ, 532, 1124

Neff, S. G., Ulvestad, J. S., \& Teng, S. H. 2004, ApJ, 611, 186

Parra, R., Conway, J. E., Diamond, P. J., et al. 2007, ApJ, 659, 314

Pérez-Torres, M. A., Mattila, S., Alberdi, A., et al. 2007, ApJ, 671, L21

Qiu, Y. L., Qiao, Q. Y., Hu, J. Y., \& Li, W.-D. 1999, IAU Circ., 7088, 2

Salpeter, E. E. 1955, ApJ, 121, 161

Sanders, D. B., \& Mirabel, I. F. 1996, ARA\&A, 34, 749

Sanders, D. B., Mazzarella, J. M., Kim, D.-C., Surace, J. A., \& Soifer, B. T. 2003, AJ, 126, 1607

Shepherd, M. C., Pearson, T. J., \& Taylor, G. B. 1995, in BAAS 27, ed. B. J. Butler, \& D. O. Muhleman, 903

Smith, H. E., Lonsdale, C. J., Lonsdale, C. J., \& Diamond, P. J. 1998, ApJ, 493, L17

Szomoru, A. 2006, in Proceedings of the 8th European VLBI Network Symposium

Ulvestad, J. S. 2009, AJ, 138, 1529

van Buren, D., Jarrett, T., Terebey, S., et al. 1994, IAU Circ., 5960, 2

Weiler, K. W., Panagia, N., Montes, M. J., \& Sramek, R. A. 2002, ARA\&A, 40, 387

Yamaoka, H., Kato, T., Filippenko, A. V., et al. 1998, IAU Circ., 6859, 1

Zezas, A., Ward, M. J., \& Murray, S. S. 2003, ApJ, 594, L31 
M. A. Pérez-Torres et al.: An extremely prolific supernova factory in the buried nucleus of the starburst galaxy IC 694, Online Material p 1

Table 1. Compact radio-emitting sources in the central region of Arp 299-A.

\begin{tabular}{|c|c|c|c|c|c|c|c|c|}
\hline \multirow{2}{*}{$\begin{array}{l}\text { Source } \\
\text { name }^{d}\end{array}$} & \multirow{2}{*}{$\begin{array}{l}\text { Source } \\
\text { type }\end{array}$} & \multirow{2}{*}{$\begin{array}{c}\Delta \alpha^{a} \\
(\mathrm{~J} 2000.0)\end{array}$} & \multirow{2}{*}{$\begin{array}{c}\Delta \delta \\
(\mathrm{J} 2000.0)\end{array}$} & \multicolumn{2}{|c|}{$S_{v}(\mu \mathrm{Jy})^{b}$} & \multicolumn{2}{|c|}{$L_{v} / 10^{26} \mathrm{erg} \mathrm{s}^{-1} \mathrm{~Hz}^{-1}$} & \multirow[t]{2}{*}{$V^{c}$} \\
\hline & & & & 8 Apr. 2008 & 5 Dec. 2008 & 8 Apr. 2008 & 5 Dec. 2008 & \\
\hline $\mathrm{A} 0$ & SN & 33.6212 & 46.707 & $318 \pm 42$ & $446 \pm 33$ & $7.9 \pm 1.0$ & $11.1 \pm 0.8$ & 2.4 \\
\hline A1 & SNR & 33.6199 & 46.699 & $855 \pm 58$ & $901 \pm 51$ & $21.3 \pm 1.4$ & $22.4 \pm 1.3$ & 0.6 \\
\hline A2 & SNR & 33.6219 & 46.655 & $708 \pm 53$ & $713 \pm 43$ & $17.6 \pm 1.3$ & $17.7 \pm 1.1$ & 0.1 \\
\hline A3 & SNR & 33.5942 & 46.560 & $398 \pm 44$ & $353 \pm 30$ & $9.9 \pm 1.1$ & $8.8 \pm 0.7$ & 0.8 \\
\hline A4 & SNR & 33.6501 & 46.537 & $558 \pm 48$ & $628 \pm 40$ & $13.9 \pm 1.2$ & $15.6 \pm 1.0$ & 1.1 \\
\hline A5 & SN? & 33.6176 & 46.693 & $278 \pm 41$ & $143 \pm 25$ & $6.9 \pm 1.0$ & $3.6 \pm 0.6$ & 2.8 \\
\hline A6 & SN & 33.6206 & 46.597 & $208 \pm 40$ & $\leq 72$ & $5.2 \pm 1.0$ & $\leq 1.8$ & 3.4 \\
\hline A7 & SNR & 33.6300 & 46.786 & $496 \pm 46$ & $468 \pm 34$ & $12.3 \pm 1.2$ & $11.6 \pm 0.8$ & 0.5 \\
\hline A8 & SNR & 33.6306 & 46.401 & $226 \pm 41$ & $264 \pm 27$ & $5.6 \pm 1.0$ & $6.6 \pm 0.7$ & 0.8 \\
\hline A9 & SNR & 33.6306 & 46.620 & $294 \pm 42$ & $282 \pm 28$ & $7.3 \pm 1.0$ & $7.0 \pm 0.7$ & 0.2 \\
\hline A10 & SNR & 33.6392 & 46.551 & $550 \pm 48$ & $436 \pm 32$ & $13.7 \pm 1.2$ & $10.9 \pm 0.8$ & 2.0 \\
\hline A11 & SNR & 33.6403 & 46.583 & $300 \pm 42$ & $351 \pm 30$ & $7.5 \pm 1.0$ & $8.7 \pm 0.7$ & 1.0 \\
\hline A12 & $\mathrm{SN}$ & 33.6495 & 46.590 & $449 \pm 45$ & $639 \pm 40$ & $11.2 \pm 1.1$ & $15.9 \pm 1.0$ & 3.2 \\
\hline A13 & SN? & 33.6531 & 46.733 & $251 \pm 41$ & $118 \pm 25$ & $6.2 \pm 1.0$ & $2.9 \pm 0.6$ & 2.8 \\
\hline A14 & SNR & 33.6825 & 46.571 & $292 \pm 42$ & $260 \pm 27$ & $7.3 \pm 1.0$ & $6.5 \pm 0.7$ & 0.6 \\
\hline A15 & $\mathrm{SN}$ & 33.5991 & 46.638 & $159 \pm 40$ & $304 \pm 28$ & $4.0 \pm 1.0$ & $7.6 \pm 0.7$ & 3.0 \\
\hline A16 & uncl. & 33.6149 & 46.516 & $\leq 117$ & $147 \pm 25$ & $\leq 2.9$ & $3.7 \pm 0.6$ & 1.2 \\
\hline A17 & uncl. & 33.6179 & 46.561 & $\leq 117$ & $179 \pm 26$ & $\leq 2.9$ & $4.5 \pm 0.6$ & 2.4 \\
\hline A18 & uncl. & 33.6192 & 46.464 & $151 \pm 40$ & $129 \pm 25$ & $3.8 \pm 1.0$ & $3.2 \pm 0.6$ & 0.5 \\
\hline A19 & SN? & 33.6196 & 46.659 & $\leq 117$ & $191 \pm 26$ & $\leq 2.9$ & $4.8 \pm 0.6$ & 2.8 \\
\hline A 20 & uncl. & 33.6278 & 46.789 & $\leq 117$ & $146 \pm 25$ & $\leq 2.9$ & $3.6 \pm 0.6$ & 1.2 \\
\hline A 21 & uncl. & 33.6291 & 46.836 & $\leq 117$ & $133 \pm 25$ & $\leq 2.9$ & $3.3 \pm 0.6$ & 0.6 \\
\hline A22 & uncl. & 33.6354 & 46.678 & $173 \pm 40$ & $217 \pm 26$ & $4.3 \pm 1.0$ & $5.4 \pm 0.7$ & 0.9 \\
\hline A23 & uncl. & 33.6360 & 46.560 & $\leq 117$ & $137 \pm 25$ & $\leq 2.9$ & $3.4 \pm 0.6$ & 0.8 \\
\hline A24 & uncl. & 33.6361 & 46.764 & $\leq 117$ & $166 \pm 25$ & $\leq 2.9$ & $4.1 \pm 0.6$ & 2.0 \\
\hline A25 & SN? & 33.6524 & 46.701 & $132 \pm 40$ & $209 \pm 26$ & $3.3 \pm 1.0$ & $5.2 \pm 0.7$ & 1.6 \\
\hline
\end{tabular}

${ }^{a}$ Coordinates are given with respect to $\alpha(\mathrm{J} 2000.0)=11: 28: 00.0000$ and $\delta(\mathrm{J} 2000.0)=58: 33: 00.000$, and were obtained from the 5 December 2008 image. The positions of those sources also detected in 8 April 2008 coincide within the errors $(\lessgtr 0.5$ mas $)$ with all of them.

${ }^{b}$ The uncertainty in the reported flux density for the detected compact components corresponds to $1 \sigma$, where $\sigma$ was determined by adding in quadrature the off-source rms in each image and a 5\% of the local maxima, to conservatively account for possible inaccuracies in the eEVN calibration.

${ }^{c}$ We define the significance of the flux density variability between the two consecutive epochs as $V=\left|S_{\text {Dec }}-S_{\text {Apr }}\right| / \sqrt{\sigma_{\text {Dec }}^{2}}+\sigma_{\text {Apr }}^{2}$, where $S_{\text {Apr }}$ and $\sigma_{\mathrm{Apr}}\left(S_{\mathrm{Dec}}\right.$ and $\left.\sigma_{\mathrm{Dec}}\right)$ are the values in Cols. 5 and 6 (7 and 8), respectively.

${ }^{d}$ Source names are given in right ascension order, except for the five components reported previously (A0 through A4) by (Neff et al. 2004). 
M. A. Pérez-Torres et al.: An extremely prolific supernova factory in the buried nucleus of the starburst galaxy IC 694, Online Material p 2

Table 2. Brightness temperatures ${ }^{a}$ of the VLBI sources in Arp 299-A.

\begin{tabular}{llllcllrr}
\hline \hline Source & \multicolumn{3}{c}{8 April 2008 } & & \multicolumn{4}{c}{5 December 2008 } \\
name & $S_{v}(\mu \mathrm{Jy})$ & $a(\mathrm{mas})$ & $b(\mathrm{mas})$ & $T_{\mathrm{B}}(\mathrm{K})$ & $S_{v}(\mu \mathrm{Jy})$ & $a(\mathrm{mas})$ & $b(\mathrm{mas})$ & $T_{\mathrm{B}}(\mathrm{K})$ \\
\hline A0 & $318 \pm 42$ & $\leq 2.8$ & $\ldots$ & $\ldots$ & $446 \pm 33$ & 5.3 & 3.0 & $1.8 \times 10^{6}$ \\
A1 & $855 \pm 58$ & $\leq 3.6$ & $\ldots$ & $\ldots$ & $901 \pm 51$ & 2.1 & 1.8 & $1.6 \times 10^{7}$ \\
$\mathrm{~A} 2$ & $708 \pm 53$ & $\leq 2.5$ & $\leq 1.5$ & $\geq 1.3 \times 10^{7}$ & $713 \pm 43$ & 2.1 & 1.6 & $1.4 \times 10^{7}$ \\
A3 & $398 \pm 44$ & $\leq 2.2$ & $\ldots$ & $\ldots$ & $353 \pm 30$ & $\leq 6.5$ & $\leq 0.3$ & $\geq 1.2 \times 10^{7}$ \\
A4 & $558 \pm 48$ & $\leq 3.6$ & $\leq 2.3$ & $\geq 1.0 \times 10^{8}$ & $628 \pm 40$ & $\leq 2.8$ & $\leq 0.5$ & $\geq 3.0 \times 10^{7}$ \\
A5 & $278 \pm 41$ & $\leq 11.1$ & $\leq 1.2$ & $\geq 1.3 \times 10^{6}$ & $143 \pm 25$ & $\ldots$ & $\ldots$ & $\ldots$ \\
A6 & $208 \pm 40$ & $\leq 7.6$ & $\leq 1.4$ & $\geq 1.3 \times 10^{6}$ & $\leq 72$ & $\ldots$ & $\ldots$ & $\ldots$ \\
A7 & $496 \pm 46$ & $\leq 2.5$ & $\leq 3.3$ & $\geq 4.0 \times 10^{6}$ & $468 \pm 34$ & 3.4 & 2.0 & $4.6 \times 10^{6}$ \\
A8 & $226 \pm 41$ & $\leq 4.8$ & $\ldots$ & $\ldots$ & $264 \pm 27$ & $\leq 3.3$ & $\leq 0.7$ & $\geq 7.6 \times 10^{7}$ \\
A9 & $294 \pm 42$ & $\leq 3.6$ & $\leq 4.4$ & $\geq 1.2 \times 10^{6}$ & $282 \pm 28$ & $\ldots$ & $\ldots$ & $\ldots$ \\
A10 & $550 \pm 48$ & $\leq 3.2$ & $\leq 3.9$ & $\geq 2.9 \times 10^{6}$ & $436 \pm 32$ & $\ldots$ & $\ldots$ & $\ldots$ \\
A11 & $300 \pm 42$ & $\leq 5.5$ & $\ldots$ & $\ldots$ & $351 \pm 30$ & $\leq 4.5$ & $\leq 1.8$ & $\geq 2.0 \times 10^{6}$ \\
A12 & $449 \pm 45$ & $\leq 3.1$ & $\leq 3.7$ & $\geq 2.6 \times 10^{8}$ & $639 \pm 40$ & 2.3 & 1.5 & $1.2 \times 10^{7}$ \\
A13 & $251 \pm 41$ & $\leq 3.3$ & $\leq 4.2$ & $\geq 1.2 \times 10^{6}$ & $118 \pm 25$ & $\leq 5.3$ & $\ldots$ & $\ldots$ \\
A14 & $292 \pm 42$ & $\leq 5.0$ & $\leq 3.7$ & $\geq 1.1 \times 10^{6}$ & $260 \pm 27$ & $\leq 4.9$ & $\ldots$ & $\ldots$ \\
A15 & $159 \pm 40$ & $\ldots$ & $\ldots$ & $\ldots$ & $304 \pm 28$ & $\leq 2.8$ & $\leq 1.6$ & $\geq 4.5 \times 10^{6}$ \\
A16 & $\leq 117$ & $\ldots$ & $\ldots$ & $\ldots$ & $147 \pm 25$ & 5.6 & 3.2 & $5.5 \times 10^{5}$ \\
A17 & $\leq 117$ & $\ldots$ & $\ldots$ & $\ldots$ & $179 \pm 26$ & 7.3 & 3.9 & $4.2 \times 10^{5}$ \\
A18 & $151 \pm 40$ & $\ldots$ & $\ldots$ & $\ldots$ & $129 \pm 25$ & $\leq 5.1$ & $\leq 5.5$ & $\geq 3.1 \times 10^{5}$ \\
A19 & $\leq 117$ & $\ldots$ & $\ldots$ & $\ldots$ & $191 \pm 26$ & $\leq 4.0$ & $\leq 2.0$ & $\geq 1.6 \times 10^{6}$ \\
A20 & $\leq 117$ & $\ldots$ & $\ldots$ & $\ldots$ & $146 \pm 25$ & $\leq 7.9$ & $\leq 3.5$ & $\geq 3.5 \times 10^{5}$ \\
A21 & $\leq 117$ & $\ldots$ & $\ldots$ & $\ldots$ & $133 \pm 25$ & $\leq 7.4$ & $\leq 2.9$ & $\geq 4.1 \times 10^{5}$ \\
A22 & $173 \pm 40$ & $\ldots$ & $\ldots$ & $\ldots$ & $217 \pm 26$ & $\leq 8.6$ & $\leq 4.3$ & $\geq 3.9 \times 10^{5}$ \\
A23 & $\leq 117$ & $\ldots$ & $\ldots$ & $\ldots$ & $137 \pm 25$ & $\leq 8.8$ & $\leq 2.9$ & $\geq 3.6 \times 10^{5}$ \\
A24 & $\leq 117$ & $\ldots$ & $\ldots$ & $\ldots$ & $166 \pm 25$ & $\leq 8.6$ & $\leq 7.0$ & $\geq 1.8 \times 10^{5}$ \\
A25 & $132 \pm 40$ & $\ldots$ & $\ldots$ & $\ldots$ & $209 \pm 26$ & $\leq 8.6$ & $\leq 4.4$ & $\geq 3.7 \times 10^{5}$ \\
\hline
\end{tabular}

${ }^{a}$ The brightness temperatures shown for the $5.0 \mathrm{GHz}$ VLBI source components in Arp 299-A were calculated using the flux densities in Table 1 and the angular sizes quoted here. We derived the brightness temperatures from the general formula: $T_{\mathrm{B}}=\left(2 c^{2} / k\right) B_{v} v^{-2}$, where $B_{v}$ is the intensity, in $\mathrm{erg} \mathrm{s}^{-1} \mathrm{~Hz}^{-1} \mathrm{str}^{-1}$. Since $B_{v}$ depends on the measured flux density, $S_{v}$, and on the deconvolved angular size of each VLBI component (obtained by fitting them to elliptical Gaussians, characterized by their major and minor semi-axis, $a$ and $b$ ). Therefore, the above formula can be rewritten as $T_{\mathrm{B}}=\left(2 c^{2} / k\right) B_{v} v^{-2}=1.66 \times 10^{9} S_{v} v^{-2}(a b)^{-1}$, where $S_{v}$ is in mJy, $v$ in GHz, and $a$ and $b$ are in milliarcseconds, respectively. 


\section{Appendix A: eEVN observations of Arp 299-A}

\section{A.1. Observing strategy}

We observed the central regions of Arp 299-A at a frequency of $5 \mathrm{GHz}$ in two epochs using the EVN. Our first epoch of 8-9 April 2008 (2008.99; experiment code RP009) included the following six antennas (acronym, diameter, location): Cambridge (CM, 32 m, United Kingdom), Medicina (MC, 32 m, Italy), Jodrell Bank (JB, 76 m, United Kingdom), Onsala (ON, $25 \mathrm{~m}$, Sweden), Torun (TO, $32 \mathrm{~m}$, Poland), and Westerbork array (WB, $25 \mathrm{~m}$, The Netherlands). Our second observing epoch on 5 December 2008 (2008.340; RP014A experiment) included, in addition the EVN antennas Effelsberg (EF, 100 m, Germany), Knockin (KN, 25 m, United Kingdom), and Shanghai (SH, 25 m, China).

Both observing epochs consisted of e-VLBI phasereferenced experiments, using a data recording rate of $512 \mathrm{Mbps}$ with two-bit sampling, for a total bandwidth of $64 \mathrm{MHz}$. The data were correlated at the EVN MkIV data processor at JIVE using an averaging time of $1 \mathrm{~s}$. First epoch observations consisted of $\sim 8.0 \mathrm{~h}$ on target. The telescope systems recorded both right-hand and left-hand circular polarization (LCP and RCP), which, after correlation, were combined to obtain the total intensity mages presented in this paper. Scans of $4.5 \mathrm{~min}$ for our target source, Arp 299-A, were alternated with 2 min scans of our phase reference source, J1128+5925. Both 3C 345 and 4C 39.25 were used as fringe finders and bandpass calibrators. Our second epoch consisted of $\sim 4.5 \mathrm{~h}$ on target. The telescope systems also recorded data in dual polarization mode, and $4.5 \mathrm{~min}$ onsource scans were alternated with 1 min scans of J1128+5925. The bright sources 3C 84, 3C 138, 4C 39.25, and 3C 286 were used as fringe finders and band-pass calibrators. We note that the inclusion of EF in the second observing epoch allowed us to achieve a much lower rms, in spite of the significantly smaller amount of total observing time.

\section{A.2. Data calibration and imaging}

We analyzed the correlated data for each epoch using the NRAO Astronomical Image Processing System (AIPS). The visibility amplitudes were calibrated using the system temperature and gain information provided for each telescope. Standard inspection and editing of the data were done within AIPS. The bandpasses were corrected using the bright calibrator 4C 39.25. We applied standard corrections to the phases of the sources in our experiment, including ionosphere corrections (using the total electron content measurements publicly available).

Because of the limited bandwidth of CM and KN, the usable data acquired by these antennas was found in a single subband with very noisy edges. To improve the quality of the bandpass calibration, we removed the edges of CM for the first epoch, and the edges of $\mathrm{CM}$ and $\mathrm{KN}$ for the second epoch. The instrumental phase and delay offsets among the 8-MHz baseband converters in each antenna were corrected using a phase calibration determined from observations of 4C 39.25. The data for the calibrator J1128+5925 were then fringe-fitted in a standard manner. We then exported the J1128+5925 data from AIPS into the Caltech imaging program DIFMAP (Shepherd et al. 1995) for mapping purposes. In this way, we determined gain correction factors for each antenna. J1128+5925 showed a flux of $\sim 0.38 \mathrm{Jy}$ at $5.0 \mathrm{GHz}$ at both epochs, and displayed a point-like structure on mas scales. After this procedure was completed within DIFMAP,

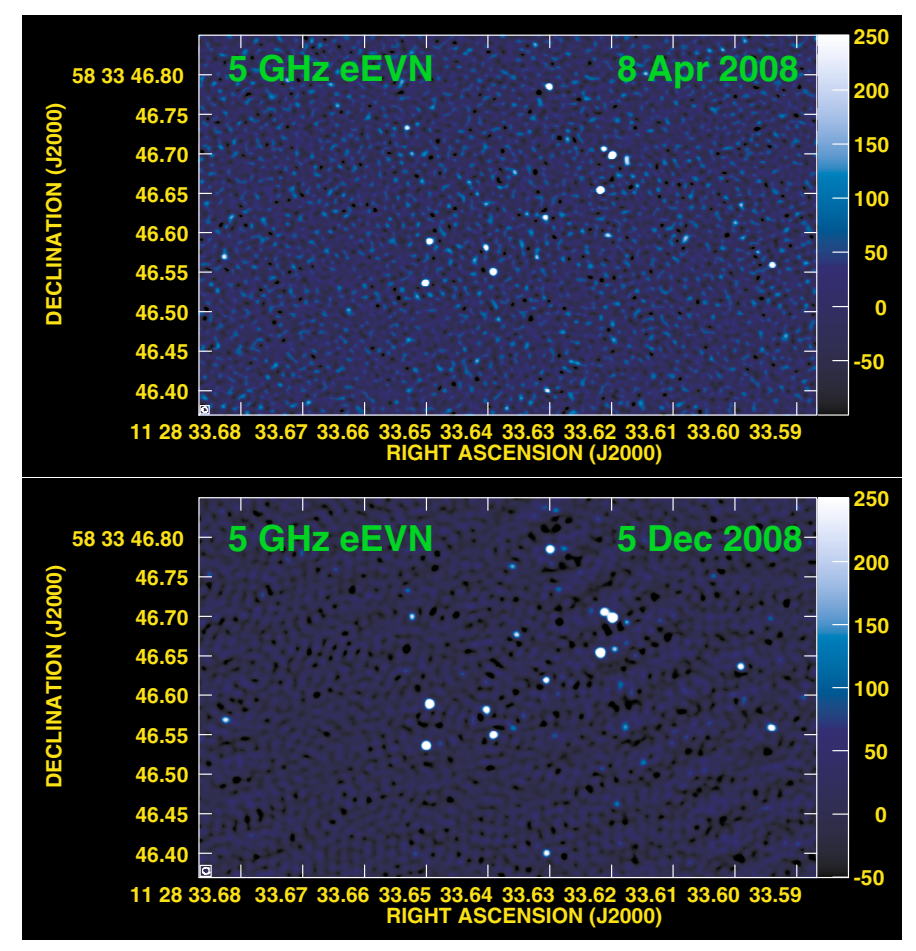

Fig. A.1. 5.0 GHz eEVN observations of the central $\sim 150$ pc years of the luminous infrared galaxy Arp 299-A on 8 April 2008 (top) and 5 December 2008 (bottom), which reveals a large population of relatively bright, compact, non-thermal emitting sources. The colour scale goes from $-50 \mu \mathrm{Jy} / \mathrm{b}$ up to $250 \mu \mathrm{Jy} / \mathrm{b}$. The size of the $F W H M$ synthesized interferometric beam was 7.3 mas $\times 6.3$ mas and 8.6 mas $\times$ 8.4 mas, respectively.

the data were read back into AIPS, where the gain corrections determined by DIFMAP were applied to the data.

The final source model obtained for J1128+5925 was then included as an input model in a new fringe-fitting search for $\mathrm{J} 1128+5925$, thus removing the structural phase contribution to the solutions of the delay and fringe rate for our target source, Arp 299-A, prior to obtaining the final eEVN images shown in Fig. 1. The phases, delays, and delay-rates determined for J1128+5925 were then interpolated and applied to the source Arp 299-A. This procedure allowed us to obtain the maximum possible accuracy in the positions reported for the compact components in Fig. 1. We note, however, that this has no impact on the final images, since $\mathrm{J} 1128+5925$ is essentially point-like at the angular resolution ( $\$ 9$ milliarcsec) provided by our 5.0 GHz e-EVN observations, and its phase-contribution is therefore negligible.

The imaging and deconvolution of Arp 299-A was performed with the AIPS task IMAGR (see Figs. 1 and A.1), using a natural weighting scheme and a ROBUST parameter equal to zero. This scheme was a good compromise when maximizing the very high angular resolution and very low rms values in our images. We point out that we kept the averaging integration time to $1 \mathrm{~s}$ and used a maximum channel bandwidth in the imaging process of $8 \mathrm{MHz}$, which results in a maximum degradation of the peak response for the component furthest away from the phase center of less than $5 \%$ and prevents artificial smearing of the images (e.g., Bridle \& Schwab 1999).

No self-calibration of the phases, nor of the amplitudes, was performed on the data, since the peaks of emission were very faint in these procedures. We emphasize two important aspects of the phase-reference technique: (i) it effectively increases the 
on-source integration time from minutes to hours, thus significantly increasing the array sensitivity, and allowing the detection and imaging of very faint objects (Beasley \& Conway 1995) and (ii) it retains the positional information about the compact components in Arp 299-A with respect to the phase-reference source $\mathrm{J} 1128+5925$, which lies $0.86^{\circ}$ from the target source.

\section{A.3. Source detection, identification, and flux density extraction}

Our 5.0 GHz eEVN observations on 8 April 2008 and 5 December 2008 resulted in off-source rms noises of $39 \mu \mathrm{Jy} / \mathrm{b}$ and $24 \mu \mathrm{Jy} / \mathrm{b}$, respectively. The difference in sensitivity was primarily due to the addition of the Effelsberg antenna in our second observing epoch, but also to there being more antennas than in our first epoch. We considered as real sources individual peaks with a signal-to-noise ratio equal to, or above five times the rms noise in either of our two observing epochs. Since our second observing epoch involved far more sensitive observations than the first one, this resulted in a larger number of detections of compact components (26) with respect to the first epoch (15).
We placed small boxes accross the 8 April 2008 image, around the positions of those components detected on 5 December 2008 , to extract the flux density of the counterparts initially detected only in the second epoch (components A15 through to A25 in Fig. 1). This procedure allowed us to recover four components above $3 \sigma$ (A15, A18, A22, and A25), based on a positional coincidence with the peak of emission in our second epoch of greater precision than 0.5 milliarcsec; and for those components not detected on 8 April, to derive $3 \sigma$ upper limits to the emission.

The flux density extraction was carried out within AIPS, using task IMFIT, which fits Gaussian components to the image. Namely, we placed small boxes (corresponding to the few inner pixels of each source) around each local peak of brightness (above five times the rms noise) and fitted single Gaussian components to each of them. Since the fitted integrated flux densities differed insignificantly from the peaks of emission (by less than $1 \%$ ), it is clear that there is no evidence that any of the compact components are resolved even with the very high angular resolution of a few milliarcsec that the eEVN delivers. The total flux densities listed in Table 1 are thus the peak flux densities found in our images. 